\title{
Dynamic Model of Per-User Service for Opportunistic Scheduling over Fading Channels
}

\author{
Mehrdad Dianati ${ }^{1}$, Rahim Tafazolli ${ }^{1}$, Xuemin Shen $^{2}$, and Kshirasagar Naik ${ }^{2}$ \\ ${ }^{1}$ Department of Electronics and Electrical Eng., University of Surrey \\ Guildford, GU2 7XH, Surrey, UK \\ ${ }^{2}$ Department of Electrical and Computer Eng., University of Waterloo \\ 200 University Ave. West, Waterloo, ON, Canada, N2L3G1
}

\begin{abstract}
In this paper, we consider maximum rate opportunistic scheduling from a single wireless base station to multiple mobile users with Rayleigh fading channels. We propose a finitestate Markovian analytical model to capture the dynamics of a single user's service, namely per-user service. The model is for a saturated scenario, where the base station always has buffered data for transmission. We demonstrate the accuracy of the model by comparing the analytical results with the results obtained from extensive simulations.
\end{abstract}

\section{INTRODUCTION}

Wireless access technologies are becoming very popular for last mile connection of Internet-enabled mobile or fixed devices. Efficient radio resource management over the last mile access networks has significant impacts on the quality of service for users and resource utilization for network operators. This is due to the fact that the shared wireless links are the main bottleneck due to certain characteristics of wireless channels. A typical radio resource management system incorporates subsystems for dynamic resource allocation, access control, and admission control. Scheduling is an efficient strategy for dynamic allocation of wireless channel resources among users with delay tolerant applications. Since the capacities of wireless access links are considerably smaller than wireline constituents of a typical connection involving service delivery from the Internet to a wireless node, it is highly desirable to deploy an efficient radio resource management system in order to ensure high spectrum utilization. Thus, modeling of the major components of a radio resource management system, in particular, a scheduler, becomes important from this perspective. Accurate modeling of a scheduling scheme is a non-trivial problem due to the complex and random nature of the key parameters such as the random behavior of channel fluctuations and a scheduling scheme itself.

In a system where a single wireless base station transmits delay tolerant data to multiple users, when the partial Channel State Information (CSI) is available, the optimal scheduling strategy to maximize spectrum efficiency is to transmit to a single user with the best channel quality in each scheduling epoch (i.e., time slot) [1], [2]. This can be considered as an opportunistic service discipline. Opportunistic scheduling with Adaptive Modulation and Coding (AMC) schemes are widely proposed for modern wireless systems [3]-[9]. Due to the wide acceptance of opportunistic scheduling, analytical modeling of its behavior has been partially considered in some existing work. In [10], a vacation-based queuing analysis for Bernoulli arrival is used to obtain delay distribution where wireless channels are independent and identically distributed Markov processes. In [12], a model for per-user throughput of an opportunistic scheduling scheme has been proposed. To the best of our knowledge, the existing publications either focus on the static behavior or per-user service or they aim to obtain certain performance metrics, e.g., throughput. Different from the existing studies, we develop a general model for the dynamic behavior of a single user's service. We demonstrate that a finite-state Markov process can adequately be used for this purpose. This conclusion is supported by the presented results of our extensive simulations. The proposed model can be used for performance analysis in radio resource management systems for the last-mile wireless access networks.

The rest of this paper is organized as follows. The important aspects of the system model are given in Section II. In Section III, we present the details of the proposed analytical model. Evaluation of the model against simulation results is given in Section IV to demonstrate the accuracy of the proposed model. Some concluding remarks are given in Section V.

\section{SySTEM MODEL}

We consider opportunistic scheduling from a single base station to multiple users, as shown in Fig. 1. An opportunistic scheduling scheme operates as follows: mobile users estimate their received Signal to Interference and Noise Ratio (SINR) from a pilot signal and report the maximum achievable rates back to the base station through a feedback channel. Comparing the received SINR of different users, the scheduler selects a single user with the highest achievable rate in each time slot. If two or more users have similar maximum achievable rates in a time slot, i.e., a tie scenario, the scheduler may apply a tie breaking policy. A simple and straightforward policy may be a random tie breaking policy that gives equal chance to all users with equal channel qualities. Mapping between the values of SINR and the achievable transmission rates is often obtained from system level simulations.

A quasi static flat fading model is assumed for the wireless fading channels, where the SINR value at a mobile station is 


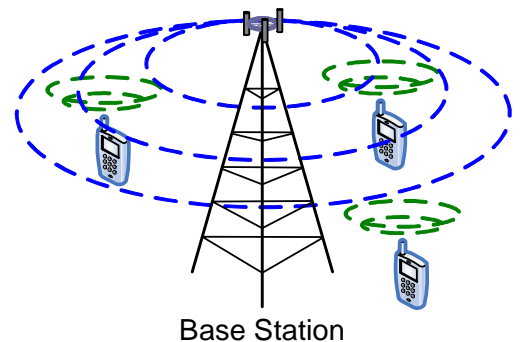

Fig. 1. System model

a random variable that remains constant for an entire duration of a time slot. In addition, we consider a finite-state Markov process, as shown in Fig. 2, to model the dynamics of the random fluctuations of the wireless channels [13]. In this model, the received SINR range is divided into multiple areas. When the signal power is below $\zeta_{i}$ and above $\zeta_{i-1}$ the channel is considered in sate $S_{i}$. The transition probability from state $S_{i}$ to state $S_{j}$ in the next time slot is denoted by $p_{i, j}$.

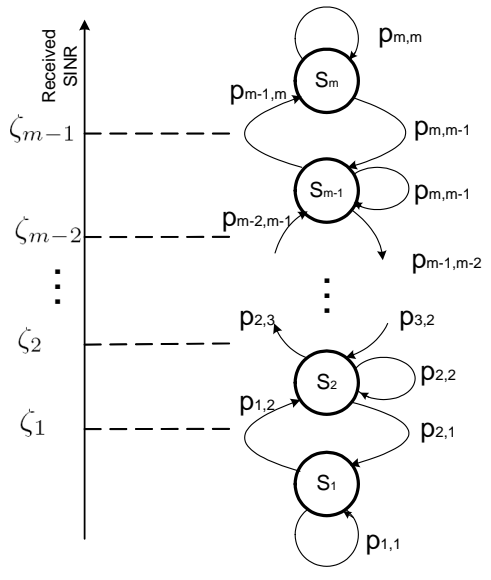

Fig. 2. Finite-state Markov model for fading channels

In the next section, we explain how the received service of a single user can be modeled in a system specified in this section.

\section{Analytical Model}

In this paper, the scheduling problem with multiple users is simplified into a scheduling problem where a tagged user competes with a single super user. The super user combines the competing capabilities of all users, except the tagged user. First, the instantaneous achievable rate of the super user is modeled by a finite-state Markov process. Next, the reduced problem is solved to obtain the service model of a single user.

\section{A. Channel Model of the Super User}

Let $N$ be the number of mobile users and $X(t)$ be the channel state of a tagged user in time slot $t$. Denote by $X_{i}(t), \quad i=1, \ldots, N-1$, the state of the channel from the base station to the compteting user $i$ in time slot $t$. The tagged user wins the competition for transmission in a time slot if $X(t)>\max \left(X_{i}(t), \ldots, X_{N-1}(t)\right)$ or when a tie is randomly broken in its favor. This competition policy suggests that the tagged user virtually competes with a super user whose channel state is given by $Z=\max \left(X_{i}, \ldots, X_{N-1}\right)$. Thus, we can simplify the analysis by replacing all competing users with a super user whose channel model can be computed from those of the $N-1$ competing users as follows.

We develop an iterative algorithm to obtain the channel model of the super user by gradual combination of the channel models of the $N-1$ competing users. First, we develop an algorithm to combine the channel models of two users. Let $p_{i, j}$ and $q_{i, j}$ be the probability of transition from state $S_{i}$ to state $S_{j}$ for users 1 and 2, respectively. Thus,

$$
\begin{gathered}
p_{i, j}=\operatorname{Pr}\left\{X_{1}(t)=S_{j} \mid X_{1}(t-1)=S_{i}\right\} \\
q_{i, j}=\operatorname{Pr}\left\{X_{2}(t)=S_{j} \mid X_{2}(t-1)=S_{i}\right\} .
\end{gathered}
$$

Denote by $Z(t)$ the channel-state of the combined super node, given by $Z(t)=\max \left(X_{1}(t), X_{2}(t)\right)$. Let $\delta_{i, j}$ be the state transition probability of $Z(t)$, defined as $\delta_{i, j}=\operatorname{Pr}\{Z(t)=$ $\left.S_{j} \mid Z(t-1)=S_{i}\right\}$. To simplify the expressions, we define the following events.

$$
\begin{aligned}
S_{i}(t): & \left\{Z(t)=S_{i}\right\} \\
S_{i, j}(t): & \left\{X_{1}(t)=S_{i}, X_{2}(t)=S_{j}\right\} .
\end{aligned}
$$

Since $Z(t)$ is the maximum of two random processes, $X_{1}(t)$ and $X_{2}(t)$, it will less frequently be in lower states. For very small values of $\operatorname{Pr}\left\{S_{i}(t-1)\right\}$, e.g., less than $0.1 \%$ of the average state probability of staying in a typical state, we can safely eliminate state $S_{i}$ and reduce the number of states. Alternatively, we can assume that the process will move to the next higher state with a probability close to 1 , i.e.,

$$
\delta_{i, j}= \begin{cases}1, & \text { if } j=i+1 \\ 0, & \text { otherwise }\end{cases}
$$

and

$$
\delta_{j, i}=0, \text { if } j<i .
$$

For significant values of $\operatorname{Pr}\left\{S_{i}(t-1)\right\}$, larger than $1 \%$ of the average value of the probability of being in any state,

$$
\begin{aligned}
\delta_{i, j} & =\operatorname{Pr}\left\{S_{j}(t) \mid S_{i}(t-1)\right\} \\
& =\operatorname{Pr}\left\{\left[\bigcup_{l=1}^{j-1}\left(S_{j, l}(t) \cup S_{l, j}(t)\right)\right] \cup S_{j, j}(t) \mid S_{i}(t-1)\right\},
\end{aligned}
$$

Since $S_{j, l}(t), S_{l, j}(t)$, and $S_{j, j}(t)$ are mutually exclusive events,

$$
\delta_{i, j}=\sum_{l=1}^{j-1}[\mu(i, j, l)+\mu(i, l, j)]+\mu(i, j, j),
$$

where

$$
\begin{aligned}
\mu(i, m, n) & =\operatorname{Pr}\left\{S_{m, n}(t) \mid S_{i}(t-1)\right\} \\
& =\frac{\operatorname{Pr}\left\{S_{i}(t-1) \mid S_{m, n}(t)\right\} \operatorname{Pr}\left\{S_{m, n}(t)\right\}}{\operatorname{Pr}\left\{S_{i}(t-1)\right\}},
\end{aligned}
$$


and

$$
\begin{aligned}
& \operatorname{Pr}\left\{S_{i}(t-1) \mid S_{m, n}(t)\right\}= \\
& \operatorname{Pr}\left\{\left[\bigcup_{k=1}^{i-1} S_{i, k}(t-1) \cup S_{k, i}(t)\right] \cup S_{i, i}(t-1) \mid S_{m, n}(t)\right\}, \\
& \operatorname{Pr}\left\{S_{i}(t-1)\right\}= \\
& \operatorname{Pr}\left\{\left[\bigcup_{k=1}^{i-1} S_{i, k}(t-1) \cup S_{k, i}(t)\right] \cup S_{i, i}(t-1)\right\}
\end{aligned}
$$

Since $S_{i, k}(t), S_{k, i}(t)$, and $S_{i, i}(t)$ are mutually exclusive events,

$$
\mu(i, m, n)=\left[\frac{T_{1}+T_{2}}{T_{3}+T_{4}}\right] \operatorname{Pr}\left\{S_{m, n}(t)\right\}
$$

where

$$
\begin{aligned}
T_{1} & =\sum_{k=1}^{i-1}\left[\operatorname{Pr}\left\{S_{i, k}(t-1) \mid S_{m, n}(t)\right\}+\operatorname{Pr}\left\{S_{k, i}(t-1) \mid S_{m, n}(t)\right\}\right] \\
T_{2} & =\operatorname{Pr}\left\{S_{i, i}(t-1) \mid S_{m, n}(t)\right\} \\
T_{3} & =\sum_{k=1}^{i-1}\left[\operatorname{Pr}\left\{S_{i, k}(t-1)\right\}+\operatorname{Pr}\left\{S_{k, i}(t-1)\right\}\right] \\
T_{4} & =\operatorname{Pr}\left\{S_{i, i}(t)\right\}
\end{aligned}
$$

Using properties of conditional probabilities,

$$
\begin{aligned}
& \operatorname{Pr}\left\{S_{i, k}(t-1) \mid S_{m, n}(t)\right\}= \\
& \frac{\operatorname{Pr}\left\{S_{m, n}(t) \mid S_{i, k}(t-1)\right\} \operatorname{Pr}\left\{S_{i, k}(t-1)\right\}}{\operatorname{Pr}\left\{S_{m, n}(t)\right\}} \\
& =\frac{p_{i, m} q_{k, n} \pi_{i, k}}{\pi_{m, n}}
\end{aligned}
$$

where

$$
\begin{aligned}
\pi_{i, k} & =\operatorname{Pr}\left\{S_{i, k}(t)\right\} \\
& =\operatorname{Pr}\left\{X_{1}(t)=S_{i}\right\} \operatorname{Pr}\left\{X_{2}(t)=S_{k}\right\} .
\end{aligned}
$$

Hence,

$$
\begin{aligned}
& \mu(i, m, n)= \\
& \frac{p_{i, m} q_{i, n} \pi_{i, i}+\sum_{k=1}^{i-1}\left(p_{i, m} q_{k, n} \pi_{i, k}+p_{k, m} q_{i, n} \pi_{k, i}\right)}{\pi_{i, i}+\sum_{k=1}^{i-1}\left(\pi_{i, k}+\pi_{k, i}\right)} .
\end{aligned}
$$

We can extend the procedure for computing the channel model of a super user to an arbitrary number of users by repeating the above algorithm, until all $N-1$ competing users are considered.

\section{B. Service Model of a Single User}

Next, the problem is reduced to a scheduling problem with two users, where the tagged user competes with a single super user. The reduced scenario can be used to obtain the service model of the tagged user. Let $X(t)$ and $Z(t)$ represent the channel states of the tagged user and the equivalent super user, respectively. Denote by $C(t)$ the service model of the tagged user. $C(t)$ can be modeled by an $(m+1)$-state Markov process, where $m$ is the number of the states of the fading channels. An extra state of $C(t)$, denoted by $S_{0}$, indicates a non-scheduled state, where the tagged user does not win the competition for transmission in time slot $t$.
First, we define the following notations:

$$
\begin{aligned}
& \gamma_{i, j}=\operatorname{Pr}\left\{C(t)=S_{j} \mid C(t-1)=S_{i}\right\} \\
& \text { Event } S_{i, j}^{\prime}(t):\left\{X(t)=S_{i}, Z(t)=S_{j}\right\} \\
& \sigma_{i, j}=\operatorname{Pr}\left\{S_{i, j}^{\prime}(t)\right\} \\
& p_{i, j}=\operatorname{Pr}\left\{X(t)=S_{j} \mid X(t-1)=S_{i}\right\} \\
& \delta_{i, j}=\operatorname{Pr}\left\{Z(t)=S_{j} \mid Z(t-1)=S_{i}\right\}
\end{aligned}
$$

To take into account the tie breaking policy, we define a binary random process, $\epsilon(t)$. When $\epsilon(t)=1$, the tagged user wins the competition; otherwise, when $\epsilon(t)=0$, the super user wins the competition. To simplify the notations, we define a new variable indicating the probability of breaking a tie in favor of the tagged user in state $S_{i}, \epsilon_{i}$, as follows:

$$
\epsilon_{i}=\operatorname{Pr}\left\{\epsilon_{i}(t)=1 \mid Z(t)=S_{i}\right\}
$$

The tie breaking policy randomly selects one of the users with the highest achievable rate that gives equal chance of winning to all users. For this policy,

$$
\epsilon_{i}=\sum_{k=1}^{N-1} \frac{1}{k+1} \operatorname{Pr}\left\{\mathrm{k} \text { competing user in state } S_{i}\right\} .
$$

$\operatorname{Pr}\left\{\mathrm{k}\right.$ competing user in state $\left.S_{i}\right\}=\left(\begin{array}{c}N-1 \\ k\end{array}\right) \pi_{i}^{k}\left(1-\pi_{i}\right)^{(N-1-k)}$,

where $\pi_{i}$ is the probability that the channel state of a single user is in state $S_{i}$. Plugging (15) into (14),

$$
\epsilon_{i}=\sum_{k=1}^{N-1} \frac{1}{k+1}\left(\begin{array}{c}
N-1 \\
k
\end{array}\right) \pi_{i}^{k}\left(1-\pi_{i}\right)^{(N-1-k)}
$$

Next, we develop an algorithm to compute $\gamma_{i, j}$. For nonsignificant values of $\operatorname{Pr}\left\{C(t-1)=S_{i}\right\}$, i.e., less than $1 \%$ of the average probability of being in any state,

$$
\gamma_{i, j}= \begin{cases}1, & \text { if } j=0 \\ 0, & \text { otherwise }\end{cases}
$$

For significant values of $\operatorname{Pr}\left\{C(t-1)=S_{i}\right\}$, we break down the problem into three separate cases: $i, j \neq 0 ; i=0, j \neq 0$; and $i \neq 0, j=0$. For $i, j \neq 0$, from definition of $\gamma_{i, j}$ in (12),

$$
\gamma_{i, j}=\operatorname{Pr}\left\{\left[\left[S_{j, j}^{\prime}(t) \cap\left(\epsilon_{i}(t)=1\right)\right] \bigcup_{k=1}^{j-1} S_{j, k}^{\prime}(t)\right] \mid C(t-1)=S_{i}\right\} .
$$

Since the right hand side of (18) is the union of mutually exclusive events,

$$
\begin{aligned}
\gamma_{i, j}= & \underbrace{\operatorname{Pr}\left\{S_{j, j}^{\prime}(t) \cap \epsilon_{i}(t)=1 \mid C(t-1)=S_{i}\right\}}_{\mathbf{A}}+ \\
& \sum_{k=1}^{j-1} \underbrace{\operatorname{Pr}\left\{S_{j, k}^{\prime}(t) \mid C(t-1)=S_{i}\right\}}_{\mathbf{B}} .
\end{aligned}
$$


Term $\mathbf{A}$ in (19) is given by

$$
\begin{aligned}
\mathbf{A} & =\operatorname{Pr}\left\{S_{j, j}^{\prime}(t) \cap \epsilon(t)=1 \mid\left[S_{i, i}^{\prime}(t-1) \cap \epsilon(t-1)=1\right] \cup\right. \\
& \left.\bigcup_{l=1}^{i-1} S_{i, l}^{\prime}(t-1)\right\} \\
= & \frac{\mathbf{A}_{\mathbf{1}}+\mathbf{A}_{\mathbf{2}}}{\mathbf{A}_{\mathbf{3}}}
\end{aligned}
$$

where

$$
\begin{aligned}
\mathbf{A}_{\mathbf{1}}= & \sum_{l=1}^{i-1} \operatorname{Pr}\left\{S_{j, j}^{\prime}(t) \cap \epsilon_{i}(t)=1 \mid S_{i, l}^{\prime}(t-1)\right\} \operatorname{Pr}\left\{S_{i, l}^{\prime}(t-1)\right\}, \\
\mathbf{A}_{\mathbf{2}}= & \operatorname{Pr}\left\{S_{j, j}^{\prime}(t) \cap \epsilon(t)=1 \mid S_{i, i}^{\prime}(t-1) \cap \epsilon(t-1)=1\right\} \times \\
& \operatorname{Pr}\left\{S_{i, i}^{\prime}(t-1) \cap \epsilon(t-1)=1\right\}, \\
\mathbf{A}_{\mathbf{3}}= & \operatorname{Pr}\left\{\left[S_{i, i}^{\prime}(t-1) \cap \epsilon(t-1)=1\right] \cup\left[\bigcup_{l=1}^{i-1} S_{i, l}^{\prime}(t-1)\right]\right\} \cdot(21)
\end{aligned}
$$

Given that

$$
\begin{aligned}
& \operatorname{Pr}\left\{S_{j, j}^{\prime}(t) \cap \epsilon(t)=1 \mid S_{i, l}^{\prime}(t-1)\right\}= \\
& \frac{\operatorname{Pr}\left\{\epsilon(t)=1 \mid S_{j, j}^{\prime}(t) \cap S_{i, l}^{\prime}(t-1)\right\} \operatorname{Pr}\left\{S_{j, j}^{\prime}(t) \cap S_{i, l}^{\prime}(t-1)\right\}}{\operatorname{Pr}\left\{S_{i, l}^{\prime}(t-1)\right\}} \\
& =\epsilon_{j} p_{i, j} \delta_{l, j},
\end{aligned}
$$

and

$$
\begin{aligned}
& \operatorname{Pr}\left\{S_{j, j}^{\prime}(t) \cap \epsilon(t)=1 \mid S_{i, i}^{\prime}(t-1) \cap \epsilon(t-1)=1\right\}= \\
& \frac{\operatorname{Pr}\left\{\epsilon(t)=1 \cap \epsilon(t-1)=1 \mid S_{j, j}^{\prime}(t) \cap S_{i, i}^{\prime}(t-1)\right\}}{\operatorname{Pr}\left\{S_{i, i}^{\prime}(t-1) \cap \epsilon_{i}(t-1)=1\right\}} \times \\
& \operatorname{Pr}\left\{S_{j, j}^{\prime}(t) \cap S_{i, i}^{\prime}(t-1)\right\} \\
& =\epsilon_{j} p_{i, j} \delta_{i, j},
\end{aligned}
$$

using (21)-(23) we can rewrite (20) as follows.

$$
\mathbf{A}=\frac{\epsilon_{j} p_{i, j} \delta_{i, j} \epsilon_{i} \sigma_{i, i}+\sum_{l=1}^{i-1} \epsilon_{j} p_{i, j} \delta_{l, j} \sigma_{i, l}}{\epsilon_{i} \sigma_{i, i}+\sum_{l=1}^{i-1} \sigma_{i, l}}
$$

Term $\mathbf{B}$ in (19) can be expanded as

$$
\begin{aligned}
\mathbf{B}= & \operatorname{Pr}\left\{S_{j, k}^{\prime}(t) \mid \bigcup_{l=1}^{i-1} S_{i, l}^{\prime}(t-1) \cup\right. \\
& {\left.\left[S_{i, i}^{\prime}(t-1) \cap \epsilon(t-1)=1\right]\right\} } \\
= & \frac{\mathbf{B}_{\mathbf{1}}+\mathbf{B}_{\mathbf{2}}}{\mathbf{B}_{\mathbf{3}}},
\end{aligned}
$$

where

$$
\begin{aligned}
\mathbf{B}_{\mathbf{1}} & =\sum_{l=1}^{i-1} \operatorname{Pr}\left\{S_{j, k}(t) \mid S_{i, l}(t-1)\right\} \operatorname{Pr}\left\{S_{i, l}^{\prime}(t-1)\right\} \\
= & \sum_{l=1}^{i-1} p_{i, j} \delta_{l, k} \sigma_{i, l} \\
\mathbf{B}_{\mathbf{2}}= & \operatorname{Pr}\left\{S_{j, k}^{\prime}(t) \mid S_{i, i}^{\prime}(t-1) \cap \epsilon(t-1)=1\right\} \times \\
& \operatorname{Pr}\left\{S_{i, i}^{\prime}(t-1) \cap \epsilon(t-1)=1\right\} \\
= & p_{i, j} \delta_{i, k} \epsilon_{i} \sigma_{i, i}, \\
\mathbf{B}_{\mathbf{3}}= & \epsilon_{i} \sigma_{i, i}+\sum_{l=1}^{i-1} \sigma_{i, l} .
\end{aligned}
$$

Hence

$$
\mathbf{B}=\frac{p_{i, j} \delta_{i, k} \epsilon_{i} \sigma_{i, i}+\sum_{l=1}^{i-1} p_{i, j} \delta_{l, k} \sigma_{i, l}}{\epsilon_{i} \sigma_{i, i}+\sum_{l=1}^{i-1} \sigma_{i, l}} .
$$

For $i=0$ and $j \neq 0$, referring to (12),

$$
\gamma_{0, j}=\operatorname{Pr}\left\{C(t)=S_{j} \mid C(t-1)=S_{0}\right\} .
$$

(20) For non-significant values of $\operatorname{Pr}\left\{C(t-1)=S_{0}\right\}$,

$$
\gamma_{0, j}= \begin{cases}1, & \text { if } j=0 \\ 0, & \text { otherwise }\end{cases}
$$

For significant values of $\operatorname{Pr}\left\{C(t-1)=S_{0}\right\}$,

$$
\begin{aligned}
\gamma_{0, j}= & \operatorname{Pr}\left\{\left[\bigcup_{k=1}^{j-1} S_{j, k}^{\prime}\right] \cup\left[S_{j, j}^{\prime} \cap \epsilon(t)=1\right] \mid C(t-1)=S_{0}\right\} \\
= & \sum_{k=1}^{j-1} \underbrace{\operatorname{Pr}\left\{S_{j, k}^{\prime}(t) \mid C(t-1)=S_{0}\right\}}_{\mathbf{D}}+ \\
& \underbrace{\operatorname{Pr}\left\{S_{j, j}^{\prime}(t) \cap \epsilon(t)=1 \mid C(t-1)=S_{0}\right\}}_{\mathbf{F}} .
\end{aligned}
$$

Term $\mathbf{D}$ in (30) is given by

$$
\begin{aligned}
\mathbf{D}= & \operatorname{Pr}\left\{S_{j, k}^{\prime}(t) \mid\left[\bigcup_{l=1}^{m} \bigcup_{n=1}^{l-1} S_{n, l}^{\prime}(t-1)\right] \cup\right. \\
& {\left.\left[\bigcup_{l=1}^{m}\left[S_{l, l}^{\prime}(t-1) \cap \epsilon(t-1)=0\right]\right]\right\} } \\
= & \frac{\mathbf{D}_{\mathbf{1}}+\mathbf{D}_{\mathbf{2}}}{\mathbf{D}_{\mathbf{3}}}
\end{aligned}
$$

where

$$
\begin{aligned}
\mathbf{D}_{\mathbf{1}}= & \sum_{l=1}^{m} \sum_{n=1}^{l-1} \operatorname{Pr}\left\{S_{j, k}^{\prime}(t) \mid S_{n, l}^{\prime}(t-1)\right\} \operatorname{Pr}\left\{S_{n, l}^{\prime}(t-1)\right\} \\
= & \sum_{l=1}^{m} \sum_{n=1}^{l-1} p_{n, j} \delta_{l, k} \sigma_{n, l} \\
\mathbf{D}_{\mathbf{2}}= & \sum_{l=1}^{m} \operatorname{Pr}\left\{S_{j, k}^{\prime}(t) \mid S_{l, l}^{\prime}(t-1) \cap \epsilon(t-1)=0\right\} \times \\
& \operatorname{Pr}\left\{S_{l, l}^{\prime}(t-1) \cap \epsilon(t-1)=0\right\} \\
= & \sum_{l=1}^{m} p_{l, j} \delta_{l, k}\left(1-\epsilon_{l}\right) \sigma_{l, l}, \\
\mathbf{D}_{\mathbf{3}}= & \operatorname{Pr}\left\{\left[\bigcup_{l=1}^{m} \bigcup_{n=1}^{l-1} S_{n, l}^{\prime}(t-1)\right] \cup\right. \\
& {\left.\left[\bigcup_{l=1}^{m}\left[S_{l, l}^{\prime}(t-1) \cap \epsilon(t-1)=0\right]\right]\right\} } \\
= & \sum_{l=1}^{m} \sum_{n=1}^{l-1} \sigma_{n, l}+\sum_{l=1}^{m}\left(1-\epsilon_{l}\right) \sigma_{l, l} .
\end{aligned}
$$

Thus,

$$
\mathbf{D}=\frac{\sum_{l=1}^{m} \sum_{n=1}^{l-1} p_{n, j} \delta_{l, k} \sigma_{n, l}+\sum_{l=1}^{m} p_{l, j} \delta_{l, k}\left(1-\epsilon_{l}\right) \sigma_{l, l}}{\sum_{l=1}^{m} \sum_{n=1}^{l-1} \sigma_{n, l}+\sum_{l=1}^{m}\left(1-\epsilon_{l}\right) \sigma_{l, l}} .
$$


Term $\mathbf{F}$ in (30) can be written as

$$
\begin{aligned}
\mathbf{F} & =\operatorname{Pr}\left\{S_{j, j}^{\prime}(t) \cap \epsilon(t)=1 \mid\left[\bigcup_{l=1}^{m} \bigcup_{n=1}^{l-1} S_{n, l}^{\prime}(t-1)\right] \cup\right. \\
& {\left.\left[\bigcup_{l=1}^{m}\left[S_{l, l}^{\prime}(t-1) \cap \epsilon(t-1)=0\right]\right]\right\} } \\
= & \frac{\mathbf{F}_{\mathbf{1}}+\mathbf{F}_{\mathbf{2}}}{\mathbf{F}_{\mathbf{3}}}
\end{aligned}
$$

where

$$
\begin{aligned}
\mathbf{F}_{\mathbf{1}}= & \sum_{l=1}^{m} \sum_{n=1}^{l-1} \operatorname{Pr}\left\{S_{j, j}^{\prime}(t) \cap \epsilon(t)=1 \mid S_{n, l}^{\prime}(t-1)\right\} \operatorname{Pr}\left\{S_{n, l}^{\prime}(t-1)\right\} \\
= & \sum_{l=1}^{m} \sum_{n=1}^{l-1} p_{n, j} \delta_{l, j} \sigma_{n, l} \epsilon_{j} \\
\mathbf{F}_{\mathbf{2}}= & \sum_{l=1}^{m} \operatorname{Pr}\left\{S_{j, j}^{\prime}(t) \cap \epsilon(t)=1 \mid S_{l, l}^{\prime}(t-1) \cap \epsilon(t-1)=0\right\} \\
& \operatorname{Pr}\left\{S_{l, l}^{\prime}(t-1) \cap \epsilon(t-1)=0\right\} \\
= & \sum_{l=1}^{m} p_{l, j} \delta_{l, j} \epsilon_{j}\left(1-\epsilon_{l}\right) \sigma_{l, l}, \\
\mathbf{F}_{\mathbf{3}}= & \operatorname{Pr}\left\{\left[\bigcup_{l=1}^{m} \bigcup_{n=1}^{l-1} S_{n, l}^{\prime}(t-1)\right] \cup\right. \\
& {\left.\left[\bigcup_{l=1}^{m}\left[S_{l, l}^{\prime}(t-1) \cap \epsilon(t-1)=0\right]\right]\right\} } \\
= & \sum_{l=1}^{m} \sum_{n=1}^{l-1} \sigma_{n, l}+\sum_{l=1}^{m}\left(1-\epsilon_{l}\right) \sigma_{l, l} .
\end{aligned}
$$

Hence,

$$
\mathbf{F}=\frac{\sum_{l=1}^{m} \sum_{n=1}^{l-1} p_{n, j} \delta_{l, j} \sigma_{n, l} \epsilon_{j}+\sum_{l=1}^{m} p_{l, j} \delta_{l, j} \epsilon_{j}\left(1-\epsilon_{l}\right) \sigma_{l, l}}{\sum_{l=1}^{m} \sum_{n=1}^{l-1} \sigma_{n, l}+\sum_{l=1}^{m}\left(1-\epsilon_{l}\right) \sigma_{l, l}} .
$$

For $i \neq 0$ and $j=0$, referring to the basic property of a transition probability matrix, i.e., the sum of each row is equal to 1 ,

$$
\gamma_{i, 0}=1-\left[\sum_{j=1}^{m} \delta_{i, j}\right]
$$

Finally, the proposed algorithm in this section for computation of $\gamma_{i, j}$ gives the transition probabilities of a mstate Markov chain which represent the dynamic behavior of received service by a single user. In the next section, we give the results of our simulation to demonstrate suitability of the model.

\section{Simulations and Evaluation of the Results}

We performed Monte Carlo simulations to verify the accuracy of the proposed analytical model. The simulation scenario includes a single base station with an arbitrary number of users, as described in Section II. For the fading channel simulator, we consider flat fading at $f_{c}=1900 \mathrm{MHz}$; the maximum Doppler shift is $10 \mathrm{~Hz}$, and the time slot duration is $1.25 \mathrm{~ms}$.

We often compare an analytically computed transition matrix, $\mathbf{P}_{\mathbf{m}}$, with the corresponding simulation result, $\mathbf{P}_{\mathbf{s}}$, for different system settings. Unlike the comparison of scalar values, matrices of arbitrary dimensions cannot be easily compared. In this paper, the average normalized norm of the rows of the error matrix, i.e., $\mathbf{P}_{\mathbf{m}}-\mathbf{P}_{\mathbf{s}}$, is used to represent the modeling error as follows.

$$
e_{m}=\frac{1}{m} \sum_{i=1}^{m} \sqrt{\frac{\sum_{j=1}^{m}\left[P_{s}(i, j)-P_{m}(i, j)\right]^{2}}{\sum_{j=1}^{m} P_{s}(i, j)^{2}}},
$$

where $m$ is the dimension of the matrices.

The summary of comparisons are shown in Figs. 3 and 4. The modeling error, defined by (38), against the normalized Doppler frequency shift is shown in Fig. 3 for $\zeta=[10,5,0] \mathrm{dB}$ (SINR partition levels in Fig. 2) and $N=10$. The normalized Doppler frequency shift represents the speed of channel fading. This figure shows that the modeling error of a 5-state Markov process for per-user service is below $2 \%$ for a good range of channel fading speed. The modeling error increases with the increasing speed of fading process due to decreasing accuracy of the underlaying channel model. Indeed, a first order Markov model becomes less accurate for fast fading channels. Figure 4 shows the per-user service model error versus the resolution of the model, i.e., the number of states in the resulting Markov process. The number of users for this simulation is $N=10$, and the maximum Doppler shift is $8.8 \mathrm{~Hz}$, which corresponds to the fading speed of a pedestrian channel. Our observation is that the modeling error for individual non-zero components of the transition probability matrix slightly increases as we increase the number of states. However, the percentage of error decreases as the number of non-zero components decreases. Figure 5 shows the modeling error against the number of users. The initial increase and the later decrease in modeling error with increasing number of users can be observed in this figure. The accuracy of the individual components of the computed probability transition matrix decreases when the number of user increases. However, when the number of users increases, the number of zero components of the matrix increases, as well. This effect decreases the modeling error according to (38).

\section{Conclusions}

In this paper, we used a finite-state Markov process to model the dynamic behavior of per-user service (service model of a tagged user) of an opportunistic scheduler for a saturated scenario, where the base station always have buffered data for transmission to the users. We tackled the problem by breaking it into two simpler problems: 1) replacing the competing users with a single super user; 2) considering an equivalent scheduling problem between the tagged user and the super user. We demonstrated that the results of the proposed model matches the simulation results with negligible approximation error. The proposed model can be used in performance analysis of resource management systems for wireless access networks, when opportunistic scheduling is deployed. For instance, the proposed model can be used for queueing analysis in order to study delay performance of opportunistic scheduling. Implementation of admission control also requires a proper service model for the underlaying scheduling scheme. Our ongoing 


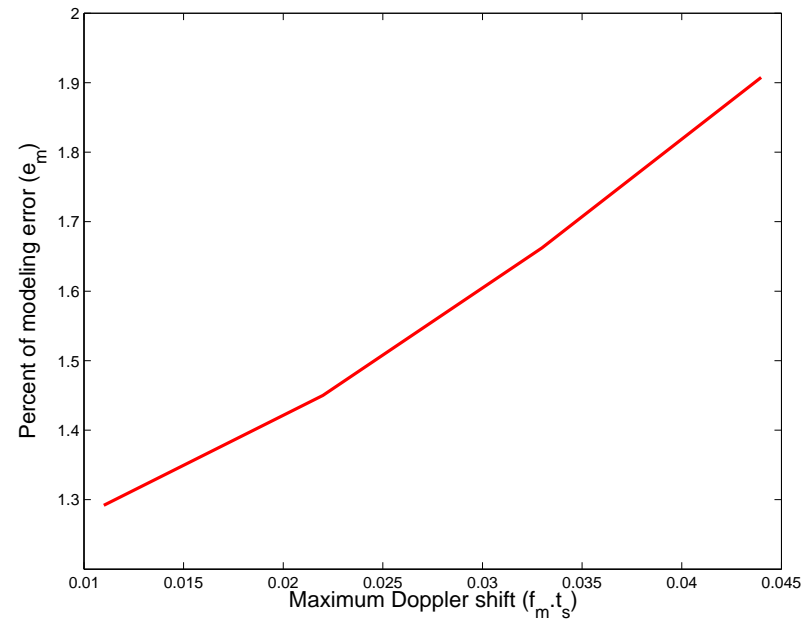

Fig. 3. Service model error vs. the speed of fading process $\left(f_{m}\right.$ is the maximum Doppler shift and $t_{s}$ is the time slot length)

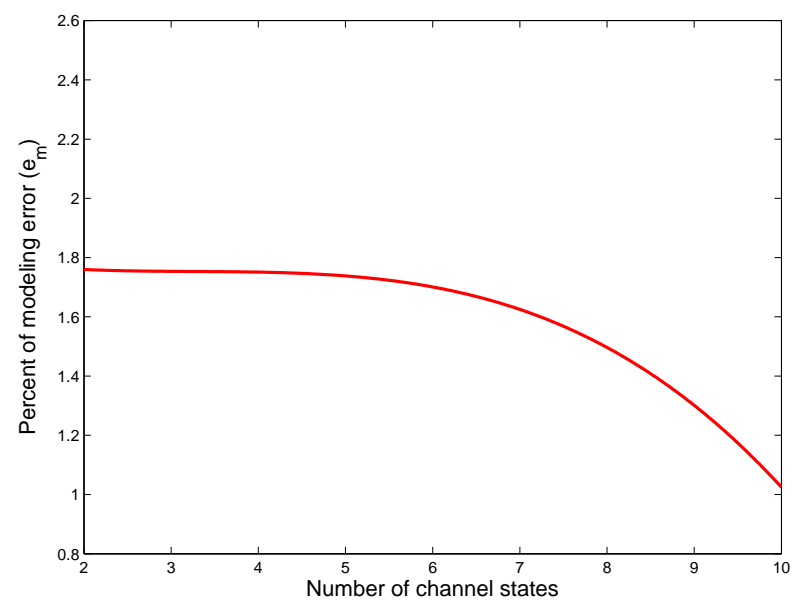

Fig. 4. Service model error vs. the resolution of the model (number of states)

work considers the extension of the model to an unsaturated case, which is more applicable. In addition, we will take into account fairness issues.

\section{ACKNOWLEDGEMENT}

This work partially supported by Research Council of United Kingdom (RCUK-EPSRC), Framework Program 7 of European Information and Communication Technologies (ICT-FP7). The work of K. Naik was partially supported by the Natural Sciences and Engineering Research Council of Canada.

\section{REFERENCES}

[1] R. Knopp and P. A. Humblet, "Information Capacity and Power Control in Single Cell Multiuser Communications," in Proc. of the IEEE Int. Conf. on Communications, June 1995, pp. 331-335.

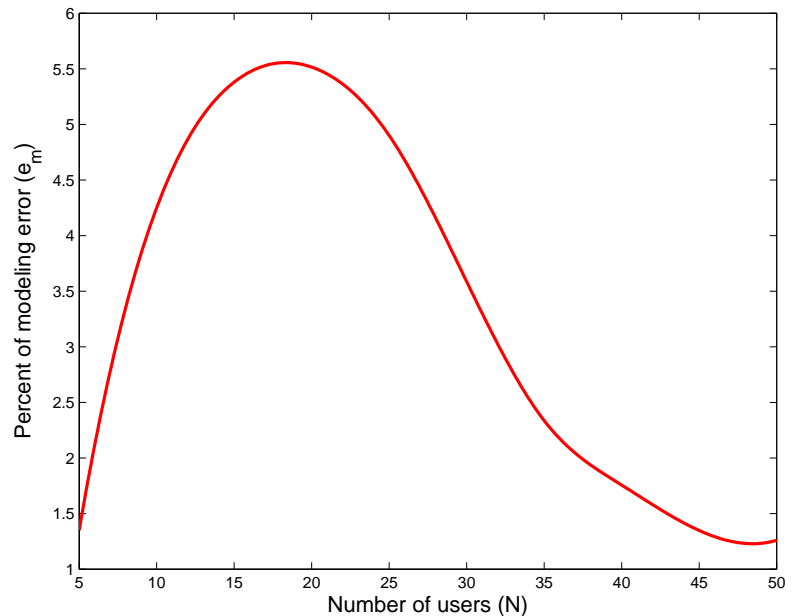

Fig. 5. Service modeling error vs. the number of users

[2] D. N. C. Tse, "Optimal Power Allocation over Parallel Gaussian Channels," in Proc. of Int. Symp. Information Theory, June 1997, pp. 27.

[3] M. Mehrjoo, M. Dianati, X. Shen, and K. Naik, "Opportunistic Fair Scheduling for the Downlink of IEEE 802.16 Wireless Metropolitan Area Networks," in Proc. of the 3rd international conference on Quality of service in heterogeneous wired/wireless networks, Waterloo, Ontario, Canada, August 2007.

[4] F. Hou, J. She, P. H. Ho, and X. Shen, "Performance Analysis on ARQ with Opportunistic Scheduling in IEEE 802.16 Networks," in Proc. IEEE GLOBECOM'07, Washington DC, USA, November 2007.

[5] R. Casaquite, K. In-Yeup Kong, Y. Myung-Hyun Yoon, and H. WonJoo, "Opportunistic Scheduling with Power Control in Ad Hoc Wireless Networks," in Proc. of The 8th International Conference on Advanced Communication Technology, vol. 1, Feb. 2006, pp. 719-724.

[6] A.K.F. Khattab, K.M.F. Elsayed, "Opportunistic Scheduling of Delay Sensitive Traffic in OFDMA-Based Wireless Network," in Proc. of International Symposium on World of Wireless, Mobile and Multimedia Networks (WoWMoM 2006), June 2006, pp. 279-288.

[7] R. Urgaonkar, M. J. Neely, "Opportunistic Scheduling with Reliability Guarantees in Cognitive Radio Networks," in proc. INFOCOM 2008, April 2008, pp. 1301 - 1309.

[8] M. Dianati, R. Tafazolli, "Opportunistic Scheduling over Wireless Fading Channels without Explicit Feedback," in proc. VTC 2008, May 2008, Singapore, pp. 1871-1875.

[9] A.K.F. Khattab, K.M.F. Elsayed, "Opportunistic Scheduling of Delay Sensitive Traffic in OFDMA-Based Wireless Network," in Proc. of International Symposium on World of Wireless, Mobile and Multimedia Networks (WoWMoM 2006), June 2006, pp. 279-288.

[10] L. B. Le, E. Hossain, and A. S. Alfa, "Delay Statistics and Throughput Performance for Multi-rate Wireless Networks Under Multiuser Diversity," IEEE Transactions $n$ Wireless Communications, vol. 5, no. 11, November 2006, pp. 3234-3243.

[11] P. Bender, P. Black, M. Grob, Robert Padovani, N. Sindhushayn, and A. Viterbi, "CDMA/HDR: A Bandwidth-Efficient High-Speed Wireless Data Service for Nomadic Users," IEEE Communication Magazine, July 2000, pp. 70-77.

[12] M. Dianati, X. Shen, and S. Naik, "Per-user Throughput of Opportunistic Scheduling in Broadcast Fading Channels," in proc. IEEE International Conference on Communications (ICC) 2006, June 2006, Istanbul, Turkey, pp. 5234 - 5239.

[13] Q. Liu, S. Zhou, and G. B. Giannakis, "Queuing With Adaptive Modulation and Coding Over Wireless Links: Cross-Layer Analysis and Design," IEEE Transactions on Wireless Communications, vol. 4, no. 3, May 2005, pp. 1142-1153. 